\section{O perfil da emergência do Hospital da Restauração: uma análise dos possíveis impactos após a municipalização dos serviços de saúde}

\section{Profile of emergency users of Hospital da Restauração: an analysis of impacts following the municipalization of healthcare}

\author{
Betise Mery Alencar S.M. Furtado \\ Hospital da Restauração \\ Secretaria Estadual de Saúde do Estado de Pernambuco \\ Universidade de Pernambuco-UPE \\ Rua Des. Célio de Castro Montenegro, 32/1101 \\ 52070-008 Monteiro/Recife/PE \\ betisemery@ig.com.br
}

José Luiz C. Araújo Jr.

Fundação Oswaldo Cruz

Centro de Pesquisas Aggeu Magalhães

\section{Paulleti Cavalcanti}

Fundação Oswaldo Cruz

Centro de Pesquisas Aggeu Magalhães

\section{Resumo}

Estudo do perfil demográfico e epidemiológico da clientela atendida na Emergência de Adultos do Hospital da Restauração, unidade de grande porte pertencente à Secretaria de Saúde de Pernambuco e que atende pacientes provenientes de todas as regiões do Estado. Objetivo: Identificar o perfil da clientela atendida, sua adequação à missão institucional e avaliar as mudanças ocorridas após a municipalização dos serviços de saúde, em 1994. Método: O desenho de estudo foi o de análise de série de dados, sendo a amostra constituída por 16.383 prontuários de pacientes atendidos nos anos de 1993, 1997 e 2001. Foram estudadas as variáveis diagnóstico (adequados e inadequados), municipalização, Programa de Saúde da Família, e as co-variáveis sexo, faixa etária, dia, hora e procedência. Resultados: Em relação à variável diagnóstico, constatou-se que a grande maioria foi inadequada $(74,5 \%)$. A municipalização dos serviços de saúde e a implantação do Programa de Saúde da Família ao que parece não acarretaram modificações significativas na demanda aos serviços. Duas faixas etárias (20-29 e 30-49 anos) perfazem mais da metade dos atendimentos. Por especialidades, a maior demanda é para a Traumatologia, seguindo-se a Clínica Cirúrgica. A quase totalidade dos pacientes (90\%) procede do Recife ou da Região Metropolitana, sendo a segunda-feira e o horário diurno os de maior demanda. Os resultados indicam que o Hospital cumpre sua missão de atendimento preferencial a politraumatismos, neurocirurgia e emergências clínicas. No entanto, atende uma grande parcela de pacientes que não estão adequados ao seu perfil, comprometendo a qualidade da assistência prestada àqueles que o demandam.

Palavras-chave: Serviços de saúde de emergência. Hospitais de emergência. Programa de Saúde da Família. Avaliação de programas. Necessidades e demandas de serviços de saúde. 


\section{Abstract}

The objective of this study is to define the demographic and epidemiologic profile of individuals who seek adult emergency care at Hospital Da Restauração, a large hospital used by people from all over the state of Pernambuco and managed by the state health department. Objective: to identify the profile of those who use the system, to assess the adequacy of the system as to institutional commitment, and to analyze the changes that have taken place following the municipalization of healthcare in 1994. Method: Analysis of a data series of a sample formed by 16,383 charts of patients who received care in 1993, 1997 and 2001. Variables studied: diagnosis (adequate and inadequate), municipalization, family health program; co-variables: gender, age group, day, time, and origin. Results: In regard to diagnoses, most were inadequate $(74.5 \%)$. The municipalization of healthcare services and the implementation of the family health program have not entailed significant changes in service demand. Two age groups make up more than half of the individuals seeking health care (20-29 and 30-39). When it comes to specialties, the greatest demand fell on trauma followed by clinical surgery. Nearly all patients (90\%) are from Recife and its surroundings. The greatest demand for healthcare takes place on Mondays and during the day. Results show that the hospital meets requirements as to providing preferential care on multiple trauma, neurosurgery and clinical emergencies. However, the fact that most cases brought to the hospital do not meet its profile compromises the quality of the care provided.

Key Words: Emergency health care services. Emergency hospitals. Family health program. Program assessments. Health care services. Needs and demands.

\section{Introdução}

O Hospital da Restauração (HR) é um hospital de grande porte, com capacidade instalada para 535 leitos. Pertence à Secretaria de Estado da Saúde do Estado de Pernambuco e está localizado em área central da cidade do Recife, facilitando o acesso a pacientes oriundos de todas as regiões da cidade e Região Metropolitana (RMR). Caracteriza-se como hospital de emergência, com uma média mensal de 800 internações, 700 cirurgias, 12.300 atendimentos ambulatoriais e 12.000 emergenciais. Tem um gasto anual médio de treze milhões de reais, excluindo a folha de pagamento dos servidores. Seu quadro de pessoal é constituído por mais de dois mil funcionários, entre nível superior, médio e elementar. A clientela atendida é universalizada e gratuita, muito variada, e não conhecida em detalhes. Estima-se que sejam atendidos indivíduos de todas as classes sociais, provenientes das mais diversas regiões do Estado.

Ao longo dos anos, o HR tem demonstrado uma marcante vocação para $\mathrm{o}$ atendimento a politraumatizados, pacientes neurológicos e casos de alta complexidade.

Não obstante sua magnitude e importância no Sistema de Saúde do Estado, ainda não está bem delineado o perfil da clientela atendida e até que ponto esta clientela corresponde e está adequada à missão do HR. Do mesmo modo, não foram ainda avaliadas as possíveis mudanças ocorridas após a municipalização dos serviços de saúde, considerando que, em 1994, o Ministério da Saúde deu início ao processo de Municipalização do Sistema Único de Saúde (SUS), reorientando o modelo assistencial a partir da assistência básica, porta de entrada do Sistema de Saúde Pública.

Estas considerações serviram de motivação para a realização do presente estudo, com o objetivo principal de conhecer e analisar o perfil demográfico e epidemiológico da clientela atendida na Emergência de Adultos do HR, no ano de 1993, comparando-o aos anos de 1997 e 2001, para verificar se houve mudança após a municipalização dos 
serviços de saúde, iniciada em 1994. Esperase que o conhecimento deste perfil possa vir a ajudar no processo de planejamento local, como também servir de estímulo à realização de estudos para o conhecimento do perfil das outras emergências da cidade, e assim contribuir para a reorganização de Rede de Saúde como um todo, atendendo o que preconiza o Art. 198 da Constituição Federal (1988), ao afirmar: "As ações e serviços de saúde integram uma rede regionalizada e hierarquizada e constituem um Sistema único" $[. . .]^{1}$.

\section{Método}

A técnica estatística utilizada para realização deste estudo foi a "análise de série de dados". A população estudada foi a clientela atendida na Emergência de Adultos do HR, nos anos de 1993, 1997 e 2001. Os dados foram coletados diretamente das fichas dos pacientes, arquivadas no Serviço de Arquivo Médico (SAME) do Hospital. A amostra foi dimensionada de forma a permitir a comparação de parâmetros referentes à demanda ao referido Serviço, nos três anos considerados, além de propiciar a comparação de parâmetros relativos a cada mês, dado o possível caráter sazonal de algumas demandas específicas. Sendo assim, considerando um volume de 12.000 atendimentos mensais e balizando o tamanho da amostra pelo objetivo de comparar pequenas proporções, decidiu-se por amostrar 480 prontuários em cada mês, de cada ano estudado. Este número foi estabelecido dimensionando-se uma amostra aleatória para estimar prevalências da ordem de $3 \%$, com erro absoluto de $1,5 \%$. Este quantitativo mensal levou a uma amostra de 5.760 prontuários para cada ano considerado, o que permitiu estimar prevalências anuais da mesma ordem, com erro absoluto menor ou igual a $0,5 \%$. A seleção da amostra foi feita de forma sistemática, utilizando-se 1 a cada 25 prontuários do SAME $(12.000 / 480=25)$, nos anos referidos, o que correspondeu a 16.383 prontuários, $27,7 \%$ referente a $1993,32,6 \%$ a 1997 e $39,7 \%$ a 2001 .
As variáveis estudadas foram: diagnósticos, municipalização, Programa de Saúde da Família, e as co-variáveis: sexo, faixa etária, dia, hora e procedência. Os diagnósticos foram classificados como "adequados e inadequados," os primeiros referentes aos pacientes que deveriam preferencialmente ser atendidos no HR, considerando sua missão, e inadequados, os diagnósticos relativos a pacientes que poderiam ser atendidos pela assistência básica. Para definir o que seria adequado e inadequado ao perfil do HR foi elaborada uma listagem com 150 diferentes diagnósticos, a partir das fichas de atendimento, e submetida a análise por uma equipe de especialistas em emergência e trauma. Dentre os diagnósticos, 40 foram considerados adequados, sendo codificados e agrupados por especialidades médicas prioritárias para atendimento no HR e, a seguir, digitados.

Os dados referentes à adesão dos municípios ao processo de municipalização e implantação do Programa de Agentes Comunitários (PACS), Programa de Saúde da Família (PSF) e tipo de gestão, referentes aos anos de 1997 e 2001, foram coletados na Secretaria Estadual de Saúde do Estado de Pernambuco.

Do total da amostra foram excluídas 723 fichas, consideradas inválidas para a análise das especialidades, por diversos motivos: não conterem dados suficientes, ou seja, fichas com diagnóstico em branco, fichas de pacientes que chegaram sem vida ao Serviço, de pessoas que desistiram do atendimento etc. Assim, para análise das especialidades, restou uma amostra de 15.660 fichas. Já para a análise de adequação e inadequação do atendimento, a amostra utilizada foi de 15.846 fichas, considerando que as pessoas que desistiram do atendimento (246 fichas) não eram casos de emergência, sendo portanto consideradas inadequadas.

\section{Plano de descrição e análise}

Os resultados foram organizados sob forma de tabelas e gráficos e analisados através de diferença de proporção, utilizando- 
se o software EPI INFO, versão 6.04. Foram feitas comparações entre os anos estudados, observando-se se houve variações no perfil da clientela atendida após a municipalização dos serviços saúde no Recife e Região Metropolitana.

Por se tratar de um estudo com dados secundários, existe o problema relativo à veracidade e qualidade das informações colhidas nos prontuários.

O estudo obteve parecer favorável da Comissão de Ética do HR, como também da Comissão de Ética do Centro de Pesquisa Aggeu Magalhães (CpqAM).

\section{Resultados e Discussão}

A partir da análise dos resultados, foi possível conhecer e avaliar os motivos que, de alguma forma, levam as pessoas a procurar a emergência do HR.

Considerando que o HR dispõe de um serviço de emergência que funciona durante 24 horas ininterruptas, com uma equipe de profissionais qualificada em várias áreas de emergências clínicas e traumáticas, acredita-se que as pessoas recorram a este serviço por razões diversas: problemas de saúde, fácil acessibilidade, funcionamento durante 24 horas, impossibilidade de recorrer aos serviços ambulatoriais e também por se encontrarem angustiadas e apreensivas.
Em relação à demanda por faixa etária (Tabela 1), duas faixas, juntas (20 a 29 e 30 a 49 anos ), concentram mais da metade dos atendimentos, nos anos estudados, coincidindo com os achados de Ribeiro ${ }^{2}$. Observou-se diferenças significativas ao comparar as prevalências por faixa etária, ao longo dos anos, em todas as faixas. Nos estudos de Salla et al. ${ }^{3}$, Deslandes et al. ${ }^{4}$, Meira et al..$^{5}$, e Horita et al. ${ }^{6}$, as faixas de maior atendimento foram-condizentes com as faixas mais prevalentes neste estudo.

Quando os resultados foram estratificados pelos anos pesquisados e relacionados a sexo e faixa etária (Tabela 2), foram constatadas associações significativas, em todos os estratos, com maior prevalência, em 1993, para homens e mulheres na faixa etária de 30 a 49 anos, e, a seguir, na faixa de 20 a 29 anos. Em 1997 e 2001 este comportamento prevalece, porém com uma maior diferença entre estas faixas e as demais; na faixa de 10 a 19 anos, a prevalência do sexo masculino é sempre superior à do feminino. Os dados relativos à faixa de 30 a 49 anos coincidem com os achados de Salla et al. ${ }^{3}$. Meira et al. ${ }^{5}$, Deslandes et al. ${ }^{4}$, Santos et al. ${ }^{7}$, e Sanchez et al. ${ }^{8}$, que constataram maioria dos atendimentos para o sexo masculino, sem correlação com a faixa etária, enquanto Horita et al. ${ }^{6}$ não encontraram diferenças importantes entre os sexos. A Tabela 3 ex-

Tabela 1 - Distribuição dos atendimentos realizados na Emergência de adultos do Hospital da Restauração, Recife, nos anos de 1993, 1997 e 2001, segundo faixa etária Table 1 - Distribution of services provided at the adult Emergency Room of Hospital da Restauração, Recife, in 1993,1997 and 2001, according to age group

\begin{tabular}{|c|c|c|c|c|c|c|c|c|}
\hline \multirow[t]{3}{*}{ Faixa etária } & \multicolumn{6}{|c|}{ Ano de referência } & \multicolumn{2}{|c|}{ Total } \\
\hline & \multicolumn{2}{|c|}{1993} & \multicolumn{2}{|c|}{1997} & \multicolumn{2}{|c|}{2001} & \multirow[b]{2}{*}{ N } & \multirow[b]{2}{*}{$\%$} \\
\hline & $\mathrm{N}$ & $\%$ & $n$ & $\%$ & $n$ & $\%$ & & \\
\hline Menor de 5 & 197 & 4,3 & 300 & 5,6 & 191 & 2,9 & 688 & 4,2 \\
\hline De 5 a 9 & 267 & 5,9 & 256 & 4,8 & 185 & 2,8 & 708 & 4,3 \\
\hline De 10 a 19 & 932 & 20,6 & 973 & 18,2 & 1013 & 15,6 & 2918 & 17,8 \\
\hline De 20 a 29 & 1184 & 26,1 & 1303 & 24,4 & 1644 & 25,2 & 4131 & 25,2 \\
\hline De 30 a 49 & 1227 & 27,1 & 1601 & 30,0 & 2243 & 34,4 & 5071 & 31,0 \\
\hline 50 ou mais & 725 & 16,0 & 906 & 17,0 & 1236 & 19,0 & 2867 & 17,5 \\
\hline Total & 4532 & 100,0 & 5339 & 100,0 & 6512 & 100,0 & 16383 & 100,0 \\
\hline
\end{tabular}


pressa os atendimentos por especialidades, em cada ano pesquisado, verificando-se proporção maior na Traumatologia, em todos os anos, com algumas variações, o mesmo ocorrendo na Clínica Médica. A Clínica Cirúrgica aparece como a terceira especialidade procurada, com um número de atendimentos relativamente estável, nos 3 anos, embora com percentuais decrescentes (Tabela 3). A Neurologia teve um comportamento constante em todos os três anos (Tabela 3). Apesar do aumento do número de atendimentos, não houve diferenças estatisticamente significativas em relação às especialidades, nos anos estudados, quando realizados os testes estatísticos.

Quando se compara os resultados de 2001 com o estudo de Meira et al.5, realizado em 1991, na mesma instituição, utilizan- do uma classificação também por especialidades, verificam-se semelhanças com os achados deste trabalho. Meira et al.5 constataram uma prevalência de $30 \%$ para a Clínica Traumatológica; na Clínica Cirúrgica, os dados foram praticamente iguais aos encontrados em 2001, ou seja, 16,35\%. Na Clínica Médica houve uma diminuição de 3,4\% no total de atendimentos, e na Neurologia um aumento de 2,4\%. Na Clínica de Otorrinolaringologia foram constatadas as maiores variações (Tabela 3). O número de atendimentos constatado neste trabalho, em 1997, foi idêntico ao encontrado por Meira et al.5 (7,3\%); quando comparado ao ano de 2001, observa-se redução de 6,0\%, possivelmente devido à transferência desta clínica para outro serviço, em 2001, deixando o HR de ser referência nesta especialidade. As especiali-

Tabela 2 - Distribuição dos atendimentos realizados na emergência de adultos do Hospital da Restauração, Recife, nos anos de 1993, 1997 e 2001, segundo sexo e faixa etária Table 2 - Distribution of services provided at the adult Emergency Room of Hospital da Restauração, Recife, in 1993, 1997 and 2001, according to gender and age group

\begin{tabular}{|c|c|c|c|c|c|c|}
\hline \multirow{3}{*}{ Faixa etária } & \multicolumn{4}{|c|}{ Sexo } & \multirow{3}{*}{$\chi^{2}$} & \multirow{3}{*}{$p$ value } \\
\hline & \multicolumn{2}{|c|}{ Masculino } & \multicolumn{2}{|c|}{ Feminino } & & \\
\hline & $\mathrm{N}$ & $\%$ & $\mathrm{n}$ & $\%$ & & \\
\hline 1993 & & & & & 24,02 & $0,0002^{*}$ \\
\hline Menor de 5 & 111 & 4,2 & 86 & 4,6 & & \\
\hline De 5 a 9 & 174 & 6,6 & 93 & 4,9 & & \\
\hline De 10 a 19 & 575 & 21,7 & 357 & 19,0 & & \\
\hline De 20 a 29 & 704 & 26,6 & 480 & 25,5 & & \\
\hline De 30 a 49 & 712 & 26,9 & 515 & 27,4 & & \\
\hline 50 ou mais & 374 & 14,1 & 351 & 18,6 & & \\
\hline 1997 & & & & & 39,81 & $0,0000^{*}$ \\
\hline Menor de 5 & 161 & 5,3 & 139 & 6,1 & & \\
\hline De 5 a 9 & 156 & 5,1 & 100 & 4,4 & & \\
\hline De 10 a 19 & 595 & 19,5 & 378 & 16,6 & & \\
\hline De 20 a 29 & 799 & 26,1 & 503 & 22,1 & & \\
\hline De 30 a 49 & 896 & 29,3 & 7,5 & 30,9 & & \\
\hline 50 ou mais & 451 & 14,7 & 455 & 20,0 & & \\
\hline 2001 & & & & & 72,52 & $0,0000^{*}$ \\
\hline Menor de 5 & 94 & 2,6 & 93 & 3,2 & & \\
\hline De 5 a 9 & 117 & 3,2 & 68 & 2,4 & & \\
\hline De 10 a 19 & 593 & 16,3 & 420 & 14,6 & & \\
\hline De 20 a 29 & 1009 & 27,8 & 635 & 22,1 & & \\
\hline De 30 a 49 & 1240 & 34,2 & 1003 & 34,9 & & \\
\hline 50 ou mais & 576 & 15,9 & 659 & 22,9 & & \\
\hline
\end{tabular}

* Associação estatisticamente significativa $(p<0,05)$ (qui-quadrado de associação)

* Statically significant association ( $p<0.05$ ) (association chi-square) 
Tabela 3 - Distribuição, por especialidades médicas, dos atendimentos realizados na Emergência de Adultos do Hospital da Restauração, Recife, nos anos de 1993, 1997 e 2001 Table 3 - Distribution of services provided at the adult Emergency Room of Hospital da Restauração, Recife, in 1993, 1997 and 2001, according to medical specialty

\begin{tabular}{|c|c|c|c|c|c|c|c|c|}
\hline \multirow[t]{3}{*}{ Especialidade } & \multicolumn{6}{|c|}{ Ano de referência } & \multicolumn{2}{|c|}{ Total } \\
\hline & \multicolumn{2}{|c|}{1993} & \multicolumn{2}{|c|}{1997} & \multicolumn{2}{|c|}{2001} & \multirow[b]{2}{*}{$\mathrm{n}$} & \multirow[b]{2}{*}{$\%$} \\
\hline & $\mathrm{N}$ & $\%$ & $\mathrm{n}$ & $\%$ & $\mathrm{n}$ & $\%$ & & \\
\hline Traumatologia & 1454 & 34,2 & 1594 & 30,5 & 2239 & 36,1 & 5287 & 33,8 \\
\hline Neurologia & 450 & 10,7 & 469 & 9,0 & 619 & 10,0 & 1538 & 9,8 \\
\hline Clínica Cirúrgica & 885 & 20,8 & 879 & 16,9 & 929 & 15,0 & 2693 & 17,2 \\
\hline Clínica médica & 1106 & 26,0 & 1188 & 22,8 & 1548 & 25,0 & 3842 & 24,5 \\
\hline Oftalmologia & 74 & 1,7 & 464 & 8,9 & 565 & 9,1 & 1103 & 7,0 \\
\hline OTL & 173 & 4,1 & 382 & 7,3 & 81 & 1,3 & 636 & 4,1 \\
\hline BMF & 52 & 1,2 & 161 & 3,1 & 131 & 2,1 & 344 & 2,2 \\
\hline Vascular & 54 & 1,3 & 76 & 1,5 & 87 & 1,4 & 217 & 1,4 \\
\hline Total & 4248 & 100,0 & 5213 & 100,0 & 6199 & 100,0 & 15660 & 100,0 \\
\hline
\end{tabular}

dades de Oftalmologia, Bucomaxilofacial e Vascular praticamente continuaram com os mesmos percentuais, nos três anos analisados. Em relação à adequação ou não do atendimento ao perfil do HR observa-se, na Tabela 4 , que, em geral, foram inadequados $(74,5 \%)$. Embora o percentual de atendimentos, no decorrer dos anos estudados, tenha aumentado em relação ao total da amostra, houve uma queda na proporção destes atendimentos inadequados (Tabela 4), demonstrando uma associação estatisticamente significativa quando realizados os testes de proporção. Segundo Peixoto Filho, Campos e Botelho $^{10}$, a presença de grande número de pacientes nos serviços de emergência de "causas comuns", neste trabalho denominados inadequados, com queixas típicas de serviços de atenção primária de saúde, caracteriza um uso abusivo destes serviços por parte da população.

Salla et al. ${ }^{3}$ encontraram $60,6 \%$ de atendimentos caracterizados como não sendo de urgência ou emergência, resultados aproximados aos achados deste estudo $(74,5 \%)$. Estes resultados demonstram claramente a utilização do HR como uma "válvula de escape” dos serviços de saúde, prejudicando o atendimento dos casos considerados adequados, uma vez que o excesso de demanda acarreta acúmulo de tarefas e conseqüente sobrecarga para toda a equipe de profissio-

Tabela 4 - Distribuição dos atendimentos realizados na Emergência de Adultos do Hospital da Restauração, Recife, nos anos de 1993, 1997 e 2001, segundo a adequação ou inadequação ao Serviço

Table 4 - Distribution of services provided at the adult Emergency Room of Hospital da Restauração, Recife, in 1993, 1997 and 2001, according to the classification of adequate or inadequate for the service

\begin{tabular}{cccccrc}
\hline \multirow{2}{*}{$\begin{array}{c}\text { Ano de } \\
\text { referência }\end{array}$} & \multicolumn{2}{c}{ Adequado } & \multicolumn{2}{c}{ Inadequado } & \multicolumn{2}{c}{ Total } \\
\hline 1993 & 1022 & 23,8 & 3276 & 76,2 & 4298 & 27,1 \\
1997 & 1372 & 26,0 & 3911 & 74,0 & 5283 & 33,3 \\
2001 & 1647 & 26,3 & 4618 & 73,7 & 6265 & 39,6 \\
Total & 4041 & $(25,5)$ & 11805 & $(74,5)$ & 15846 & 100,0 \\
\hline
\end{tabular}

$\chi^{2}=9,37$ (qui-quadrado de associação) (association chi-square); $\mathrm{p}=0,009$ 
nais, contribuindo também para o aumento dos custos hospitalares. Por outro lado, o fato também aponta para a ineficácia do Sistema de Saúde, incapaz de oferecer a esses indivíduos um acesso mais fácil à rede de atenção primária, encaminhando-os aos serviços de urgência e emergência. Observouse redução nos atendimentos a pacientes procedentes do Recife e aumento nos procedentes dos municípios da RMR e outras localidades. No Recife, os atendimentos passaram de 51,1\% em 1993 para $45 \%$ em 1997, e $44,4 \%$ em 2001, enquanto na RMR, responsável por $38,7 \%$ da demanda, em 1993, os atendimentos aumentaram para $44,9 \% \mathrm{em}$ 2001. O que chama a atenção é o fato do Recife e da RMR serem responsáveis por quase $90 \%$ dos atendimentos, restando apenas $10 \%$ para as outras regiões do Estado e fora dele. Considerando que o Serviço é para todo o Estado, extrapolando os limites territoriais entre municípios, é de se questionar as razões para esta reduzida procura por parte da população do interior, levando-se em consideração que os atendimentos de quase metade da amostra são para pacientes da cidade do Recife. Poder-se-ia inferir que a população interiorana não está sujeita a urgências médicas com a mesma freqüência que aqueles que moram numa cidade grande, que a menor densidade populacional faria diminuir naturalmente $o$ risco de adoecer, ou ainda que esta população estaria sendo atendida por outros serviços, tanto hospitais regionais como outros grandes hospitais da cidade do Recife. Quando se relaciona a procedência com os distintos anos do estudo, verifica-se uma relação estatisticamente significativa, $\operatorname{com} \mathrm{p}<0,05$.

Em relação à inadequação dos atendimentos, por município da RMR, pode-se observar, na Tabela 5, uma oscilação muito grande em quase todos os municípios quando se compara os três anos entre si, o que dificulta a análise, uma vez que apenas dois municípios mantiveram uma diminuição do número de casos inadequados, de forma consecutiva - Itamaracá e Jaboatão - enquanto em Ipojuca, Itapissuma e Paulista ocorreu um aumento dos casos inadequa- dos. Nos municípios do Recife, Olinda, São Lourenço da Mata, Abreu e Lima e Moreno houve aumento nos atendimentos em 1997, quando comparado a 1993, e uma diminuição em 2001. Em Araçoiaba, Cabo, Camaragibe e Igarassu os atendimentos diminuíram em 1997 e aumentaram em 2001. Se a comparação for apenas entre os anos de $2001 \mathrm{e}$ 1993, em nove municípios houve diminuição dos casos inadequados. Vale ressaltar que, nos municípios de maior porte, também ocorreu diminuição dos casos inadequados (Tabela 5). O que se pode inferir, a partir destes resultados, é que, em 1997, muitos municípios ainda estavam iniciando o processo de municipalização, e mesmo aqueles que já o adotavam desde 1994 ou 1995, ou já tinham implantado o PSF, ainda contavam com uma cobertura incipiente, não causando impacto na situação de saúde. Já em 2001 (Tabela 5), observa-se maior uniformidade na diminuição dos atendimentos inadequados nestes municípios, possivelmente por conta da melhoria da assistência básica. O município de Camaragibe chama a atenção por ter sido um dos primeiros a implantar o PSF, com melhoria em vários indicadores de saúde, e por apresentar, neste estudo, diminuição do número de casos inadequados em 1997 (2,5\%), quando comparado a 1993, e um aumento destes casos em 2001. Farias ${ }^{12}$ afirma que o modelo de atenção, naquele município, é básico-centro, não estando estabelecida a regionalização por níveis de complexidade, não havendo um sistema de referência e contrareferência formal, e preponderando a referência informal envolvendo serviços fora do município. $\mathrm{O}$ que se pode deduzir, a partir daí, é que o Programa de Saúde da Família, como proposta de "porta de entrada" do Sistema de Saúde, “[...] não tenha poder para reverter a configuração do modelo médico hegemônico" ${ }^{13}$. D'Aguiar ${ }^{14}$, em Volta Redonda, concluiu que há uma precária integração com a rede local de saúde, o que contribui para a formação de demanda espontânea por parte daqueles que não conseguem resolver seus problemas de saúde na rede básica, como preconiza o Ministério da Saúde. 
Deste modo, o PSF deixa de ser a "porta de entrada" do Sistema e não são utilizados os hospitais para os atendimentos mais complexos e que requeiram maior tecnologia ${ }^{15,16}$.

Quando se faz a relação do tipo de gestão, baseado nas Normas Operacionais Básicas (NOBs - 93 e 96) dos municípios da RMR e a cobertura do PSF, com a diminuição dos casos inadequados na Emergência de Adultos do HR (Tabela 5), verifica-se não existir associação no ano de 1997, como referido anteriormente, provavelmente pelo incipiente grau de comprometimento dos municípios com o processo de municipalização e pela baixa cobertura do PSF. Já em 2001, a análise torna-se mais plausível, observandose diminuição dos casos inadequados, de forma mais uniforme, entre os municípios, em comparação a 1993 (anterior à implantação do PSF). Dos 14 municípios, apenas em 4 não diminuiu o número de casos inadequados no período estudado: Abreu e Lima e Itapissuma, embora contassem com cobertura do PSF acima de 50,0\% e estivessem no Sistema de Gestão Plena da Atenção Básica; Ipojuca, com uma cobertura de 18,75\% e Gestão Plena da Atenção Básica; e Paulista, com uma cobertura do PSF de 30,10\% e Gestão Plena do Sistema Municipal.

Segundo o Ministério da Saúde ${ }^{15}$, os principais benefícios para os municípios em que estão implantados o PACS e o PSF são "a melhoria dos indicadores de saúde, diminuição do número de exames complementares, de encaminhamentos de emergência e de internações hospitalares". Segundo ainda o $\mathrm{MS}^{9}$, o avanço dos municípios em relação ao sistema de gestão acarretou maiores responsabilidades para eles e para os gestores

Tabela 5 - Proporção dos municípios da Região Metropolitana com o tipo de gestão e cobertura do PSF, em 1997 e 2001, comparados ao ano de 1993, quanto à proporção de casos inadequados atendidos na Emergência de Adultos do Hospital da Restauração, Recife

Table 5 - Proportion of municipalities within the Metropolitan Area according to type of management and coverage of the PSF, in 1997 and 2001, in comparison to 1993 as to the ratio of inadequate cases dealt with at the adult Emergency Room of Hospital da Restauração in Recife

\begin{tabular}{|c|c|c|c|c|c|c|c|}
\hline \multirow[b]{3}{*}{ MUNICÍPIOS } & \multicolumn{7}{|c|}{ Ano de referência } \\
\hline & \multirow{2}{*}{$\begin{array}{c}1993 \\
\% \text { de } \\
\text { Inadequados }\end{array}$} & \multicolumn{2}{|l|}{1997} & \multicolumn{4}{|c|}{2001} \\
\hline & & $\begin{array}{c}\text { Tipo de gestão } \\
\text { s NOB/93 In }\end{array}$ & $\begin{array}{c}\% \text { de } \\
\text { nadequados }\end{array}$ & $\begin{array}{c}\text { Tipo de gestão } \\
\text { NOB/96 }\end{array}$ & $\begin{array}{l}\text { Número de } \\
\text { equipes PSF }\end{array}$ & $\begin{array}{c}\text { \% de } \\
\text { cobertura PSF }\end{array}$ & $\begin{array}{c}\% \text { de } \\
\text { Inadequados }\end{array}$ \\
\hline Abreu e Lima & 60,9 & Incipiente & 75,4 & PAB & 17 & 57,97 & 74,5 \\
\hline Araçoiaba & 83,3 & - & 55,5 & PAB & 04 & 53,19 & 77,8 \\
\hline Cabo & 61,1 & Incipiente & 54,7 & PSM & 32 & 55,99 & 57,4 \\
\hline Camaragibe & 67,0 & Parcial/Semi-plena & 64,5 & PSM & 31 & 95,79 & 65,1 \\
\hline Igarassu & 70,2 & Incipiente & 59,7 & PAB & 16 & 78,14 & 69,7 \\
\hline Ipojuca & 42,3 & - & 50,0 & PAB & 04 & 18,75 & 54,3 \\
\hline Itamaracá & 100,0 & Incipiente & 80,0 & PAB & 03 & 50,96 & 62,5 \\
\hline Itapissuma & 55,5 & Parcial & 63,6 & PAB & 04 & 67,21 & 90,0 \\
\hline Jaboatão & 71,4 & Incipiente & 68,7 & PAB & 23 & 13,72 & 67,5 \\
\hline Moreno & 63,6 & Incipiente & 70,6 & PSM & 06 & 37,36 & 54,5 \\
\hline Olinda & 74,9 & Parcial/Semi-plena & 77,9 & PSM & 33 & 39,62 & 73,8 \\
\hline Paulista & $67,0 \quad \ln$ & ncipiente/Semi-plena & 74,7 & PSM & 26 & 30,10 & 78,5 \\
\hline Recife & 76,9 & Incipiente & 77,4 & PSM & 82 & 14,59 & 74,8 \\
\hline $\begin{array}{l}\text { São Lourenço } \\
\text { da Mata }\end{array}$ & 50,9 In & ncipiente/Semi-plena & 73,9 & PAB & 22 & 36,05 & 61,6 \\
\hline
\end{tabular}

FONTE. Ministério da Saúde e IBGE- Pacto de atenção básica-2002. PAB - Plena de Atenção Básica / PSM - Plena do Sistema Municipal/SES-2003. PSF- Programa de Saúde da Família. NOB - Norma Operacional Básica

SOURCE. Ministry of Health and IBGE - Basic Care Pact - 2002. PAB - Full Basic Care / PSM - Full Municipal System/SES-2003. PSF - Family Health Program. NOB Basic Operational Standard 
municipais, como também aumento da eqüidade dos recursos financeiros, por meio da implantação do Piso Assistencial Básico (PAB), mas não garantiu a qualidade nem a efetividade da atenção básica nem o acesso dos cidadãos aos outros níveis de atenção. É o que se pode constatar, através dos dados apresentados na Tabela 5. Dos seis municípios com Gestão Plena do Sistema Municipal, Moreno foi o que apresentou maior diminuição de casos inadequados $(9,1 \%)$, seguido do Cabo $(3,7 \%)$ e do Recife $(2,1 \%)$. Nos outros dois, Camaragibe e Olinda, a redução foi inferior a 2,0\%. Em Paulista os casos inadequados não diminuíram; pelo contrário, aumentaram $11,5 \%$.

Poucos são ainda os trabalhos referentes à atuação do PSF, voltados para a avaliação de sua função maior, de reordenador de demanda da atenção básica; o que há são trabalhos voltados para a avaliação administrativa, realizados pelo próprio Ministério da Saúde. D'Aguiar ${ }^{14}$, ao avaliar a resolubilidade do Programa, chega à conclusão de que “... o Programa de Saúde da Família, em Volta Redonda, ainda não conseguiu incorporar o novo paradigma da promoção da saúde [...] e continua concentrando a sua atuação na resolução dos casos de doença que acorrem aos módulos e implementação de atividades enquadradas nos programas considerados prioritários pelo Ministério da Saúde [...] mas a própria assistência às doenças é de qualidade insatisfatória".

\section{Considerações Finais}

O estudo se reveste de fundamental importância, do ponto de vista do pesquisador, uma vez que a literatura não registra, em Pernambuco, nenhuma pesquisa com a amplitude aqui desenvolvida, e sobretudo em relação ao objeto do estudo, o HR, instituição de tanta importância para a população pernambucana, não somente em relação à assistência prestada, mas também ao seu papel e real significação na história recente da saúde no Estado.

Conhecer o perfil da clientela que utiliza o HR foi o objetivo maior do trabalho, para servir de ponto de apoio e facilitador no planejamento das ações dos gestores.

Os resultados permitem caracterizar a Emergência de Adultos do HR em relação às faixas etárias que a utilizam, horários mais procurados, procedência dos pacientes e diagnósticos; além disso, tenta-se estabelecer uma ponte entre estes diagnósticos e a assistência prestada pela Rede Básica de Saúde, inferindo se ocorreram mudanças no perfil do atendimento a partir da implementação de políticas públicas definidas como prioritárias para esta área, como é o caso do Programa da Saúde da Família.

O que se pode observar, através dos resultados e após a realização de testes estatísticos para avaliar a significância das associações, é que a faixa etária que mais demandou a Emergência foi a de 20 a 49 anos, a maioria do sexo masculino, procedente, em quase $90 \%$ dos casos, do Recife e RMR, mais da metade residente no Recife, fato que chama a atenção.

Os dados coletados levam a inferir que o $\mathrm{HR}$, mais do que se poderia imaginar, atende prioritariamente a população da capital, desde à atenção básica, para uma grande parcela, até os procedimentos mais complexos, fato que leva a outra inferência: o HR não está tão voltado para a demanda procedente do interior, conforme se imaginava.

Em relação à inadequação do atendimento, o HR supera o que se encontrou na literatura, atingindo um percentual, relativo aos três anos estudados, de 74,5\%, acima do encontrado no estudo de Farjado Ortiz e Ramírez-Fernandez ${ }^{11}$, de $64,1 \%$, e de Sanchez et al. ${ }^{8}$, de $36,9 \%$.

Para o elevado número de pacientes com atendimentos considerados inadequados, poder-se-ia levantar uma série de causas explicativas: interesse dos hospitais-escola em garantir elevado número de atendimentos, assegurando assim a fonte de internação, como também a permanência como local de experiência para os estudantes; manutenção do fluxo financeiro, através do sistema de pagamento por consulta; acesso mais fácil do que o serviço de atenção primária; suposição, por parte da população, de que o 
médico é mais experiente; desinformação da população sobre o funcionamento dos serviços de emergência; consultas negadas na assistência primária; maior longevidade da população, aumentando o número de idosos nos serviços de emergência, o mesmo acontecendo com o número de consultas por afecções leves e crônicas.

O HR apresenta muitas características similares a estas, comprovadas pelo elevado número de casos inadequados encontrados no estudo. Sendo um hospital de ensino, muitas vezes o interesse de quem atende (médico) não é o mesmo da instituição, ocasionando um descompasso no decorrer das atividades do dia-a-dia na unidade. No que concerne à população, é patente o baixo nível de informação, ou seja, a desinformação sobre o funcionamento dos serviços de saúde, claramente demonstrada no período de carnaval, quando há um redirecionamento dos atendimentos, por determinação das autoridades, e se verifica uma queda no número de casos atendidos na unidade.

Quando se analisa os atendimentos adequados, constata-se que apenas $25,5 \%$ do total foram assim classificados neste estudo. Esta classificação tomou por base os diagnósticos que deveriam preferencialmente procurar o $\mathrm{HR}$, em suas várias especialidades, e a missão institucional.

Dentre as clínicas, os resultados reforçam a característica principal do HR, que é atender os politraumatismos, confirmandose a predominância da neurocirurgia, dos traumas de crânio e, na clínica cirúrgica, das lesões penetrantes e politraumas.

Quanto ao mês de atendimento, dia da semana e hora, há um certo equilíbrio nos meses do ano, com uma queda em fevereiro, coincidente com o período de carnaval. Em relação ao dia da semana, comprovou-se a suspeita, através da observação empírica, de ser a segunda feira o dia de maior movimento, o que pode ser explicado pela falta de alternativas de serviços de atenção básica funcionando durante o final de semana, ocasionando assim um acúmulo de atendimento neste dia. Em relação aos horários, a maioria dos atendimentos acontece durante o dia, tanto os adequados como os inadequados.

A partir destas elucidações, é possível apontar o HR como "porta de entrada" do Sistema de Saúde Pública, contrariamente ao objetivo do Ministério da Saúde, ao preconizar que a "porta de entrada" do Sistema deve ser a rede básica, principalmente o PSF. Ainda é cedo para qualquer conclusão mais profunda e segura a respeito deste propósito, uma vez que a cobertura do PSF, na maioria dos municípios, ainda é incipiente. No entanto, vale ressaltar que o município de Camaragibe, com uma cobertura de mais de $90 \%$ do PSF, contribui com $65,1 \%$ de casos considerados inadequados para a emergência do HR. São paradoxos difíceis de explicar.

Extrapolando o contexto do estudo, o que se pode deduzir é que os serviços de emergência devem ser sistematicamente avaliados para se acompanhar sua evolução, adequação às políticas implementadas e se estas satisfazem os anseios e são capazes de solucionar as necessidades da população em relação à problemática de saúde.

Poder-se-ia definir este estudo como um esforço para começar a desvendar, de uma forma mais global, o Hospital da Restauração. Novos projetos deverão surgir para melhor se conhecer os vários aspectos que envolvem o seu funcionamento e traçar um perfil institucional que abranja outros enfoques, como custos e resolubilidade.

\section{Referências}

1. Brasil. Ministério da Saúde. Secretaria Nacional de Assistência à Saúde. Sistema Único de Saúde. Brasília; 1998.

2. Ribeiro CA. O padrão de resolutividade do Pronto Socorro Municipal da Lapa, São Paulo.[dissertação de mestrado]. São Paulo: Universidade de São Paulo; 1998.
3. Salla J, Chellar ML, Ilha MS, Frandoloso PR. Perfil da demanda de um serviço de Pronto Socorro, Santa Maria, RS. Saúde 1989; 15(1/2): 71-80.

4. Deslandes S, Silva C. Análise da morbidade hospitalar por acidentes de trânsito em hospitais públicos do Rio de Janeiro, RJ, Brasil. Rev Saúde Pública 2000; 34(4): 367-72. 
5. Meira MRL, Evangelista J, Burgos AR, Quitute A, Olinda H, Olegário FJ. Hospital da Restauração: análise do atendimento na emergência de adultos. Revista do Hospital da Restauração 1992; 3: 35-7.

6. Horita R, Oliveira E, Cardoso N, Oliveira R, Costa R. Características clínico epidemiológicas dos atendimentos do Pronto Socorro Psiquiátrico do Hospital São Vicente de Paulo em 1995, 1996. Revista de Saúde do Distrito Federal 1996; 7(3): 35-9.

7. Santos ME, Amor JA, Del-Bem C, Zuadi AW. Serviço de emergências psiquiátricas em hospital geral universitário: estudo prospectivo. Rev Saúde Pública 2000; 34(5): 468-74.

8. Sanchez JC, Toledano MCC, Mendoza HDF, Ortiz GF. Perfil de las demandas de atención médica en el servicio de urgencia: 9,515 casos. Cirurgía y Cirujanos 1998; 66(1): 21-3.

9. Brasil. Ministério da Saúde. Programa Saúde da Família chega a mais 219 municípios. Notícias, 19 de março de 2002. Disponível em: <http:// portalweb02.saude.gov.br /saude/aplicacoes/.../ noticias_detalhe.cfm?co_seq_noticia=283>.

10. Peixoto Filho AJ, Campos HD, Botelho LJ. Serviço de emergência em clínica médica do Hospital da UFSC: estudo do perfil da demanda. Arquivos Catarinenses de Medicina 1990; 19(1): 37-44.

11. Farjado Ortiz G, Ramirez-Fernandez F. Utilización del servicio de urgencias en un hospital de especialidades. Cirurgia y Cirujanos 2000; 68(4): 164-8.
12. Farias SF. Modelo assistencial e responsabilidade sanitária: os limites e possibilidades da atenção à gestante no Município de Camaragibe-Pernambuco. [dissertação de mestrado]. Recife: Centro de Pesquisas Aggeu Magalhães, Fundação Oswaldo Cruz; 1998.

13. Franco T, Merfhy E. PSF: contradições e novos desafios. In: 10a Conferência Nacional de Saúde On Line; 1996: Tribuna livre. Brasília. 1997. Disponível em: <www.datasus.gov.br/cns/temas/ tribuna/ PsfTito.htm>.

14. D’Aguiar J M M. O Programa Saúde da Família no Brasil. A Resolutividade do PSF no Município de Volta Redonda (RJ) [tese de doutorado]. Rio de Janeiro: Escola Nacional de Saúde Pública, Fundação Oswaldo Cruz, 2001.

15 Brasil. Ministério da Saúde. Secretaria de Políticas de Saúde. Programa Saúde da Família: Informes Técnicos Institucionais. Rev Saúde Pública 2000; 34(3): 316-9.

16. Brasil. Ministério da Saúde. Anuário estatístico de saúde do Brasil- 2001. Brasília; 2001.Disponível em: http://portal.saude.gov.br/saude/aplicacoes / anuario2001morb/introd.cfm $>$.

recebido em: 23/03/04 versão reformulada apresentada em: 18/06/04 aprovado em: 02/07/04 